EUROPEAN ORGANIZATION FOR NUCLEAR RESEARCH

CERN - AB Division

CERN-AB-2003-011 ABP

CLIC Note 562

Colliding Nanobeams in CLIC with Magnets Stabilized to the Sub-nm Level

\author{
R. Assmann, W. Coosemans, G. Guignard, S. Redaelli, \\ D. Schulte, I. Wilson, F. Zimmermann, CERN
}

The Compact Linear Collider (CLIC) aims at colliding $\mathrm{e}^{\wedge}+\mathrm{e}^{\wedge}$ - beams at 1:5 TeV with effective transverse spot sizes of $60 \mathrm{~nm}$ (horizontal) times $0: 7 \mathrm{~nm}$ (vertical). Strict stability tolerances must be respected in order to achieve a sufficient overlap of the two colliding beams. A stability test stand has been set up at CERN, bringing latest stabilization technology to the accelerator field. Using this technology, a CLIC prototype magnet was stabilized in a normal CERN working environment to less than 1-nm vertical RMS motion above $4 \mathrm{~Hz}$. Detailed simulations of the time-dependent luminosity performance of CLIC are discussed. They include the beam-beam interaction, the beam-based feedbacks and the measured data on magnet stability

\author{
Presented at PAC 2003, Portland, Oregon, USA \\ from 12 to 16 May 2003
}

Geneva, Switzerland

22 May 2003 


\title{
Colliding Nanobeams in CLIC with Magnets Stabilized to the Sub-nm Level
}

\author{
S. Redaelli*, R. Aßmann, W. Coosemans, G. Guignard, \\ D. Schulte, I. Wilson, F. Zimmermann, CERN, Geneva, Switzerland
}

\begin{abstract}
The Compact LInear Collider (CLIC) aims at colliding $\mathrm{e}^{+} \mathrm{e}^{-}$beams at $1.5 \mathrm{TeV}$ with effective transverse spot sizes of $60 \mathrm{~nm}$ (horizontal) times $0.7 \mathrm{~nm}$ (vertical). Strict stability tolerances must be respected in order to achieve a sufficient overlap of the two colliding beams. A stability test stand has been set up at CERN, bringing latest stabilization technology to the accelerator field. Using this technology, a CLIC prototype magnet was stabilized in a normal CERN working environment to less than 1-nm vertical RMS motion above $4 \mathrm{~Hz}$. Detailed simulations of the timedependent luminosity performance of CLIC are discussed. They include the beam-beam interaction, the beam-based feedbacks and the measured data on magnet stability.
\end{abstract}

\section{INTRODUCTION}

The Compact LInear Collider (CLIC) Study at CERN [1] aims at colliding $\mathrm{e}^{+} \mathrm{e}^{-}$beams at $1.5 \mathrm{TeV}$ with a luminosity of about $10^{35} \mathrm{~cm}^{-1} \mathrm{~s}^{-1}$. In order to achieve this luminosity performance the transverse beam size at the interaction point must be as small as $60 \mathrm{~nm}$ (horizontal) times $0.7 \mathrm{~nm}$ (vertical). CLIC will rely on a beam based feedback system for the correction of magnet motion below few $\mathrm{Hz}$, but the faster motion of the quadrupole magnetic center must be mechanically stabilized to a fraction of the beam size. The vertical uncorrelated motion above $4 \mathrm{~Hz}$ for the CLIC quadrupoles must be smaller than $1.3 \mathrm{~nm}$ (linac) and $0.2 \mathrm{~nm}$ (final doublets) [2]. The final goal for the CLIC Stability Study is to demonstrate that such tolerances can actually be met. The approach that has been followed is to bring modern stabilization technologies to the accelerator field. The goals of the first phase of the CLIC Stability Study, as defined in January 2002, are: (1) Establish vibration measurements with sub-nanometer accuracy; (2) Investigate modern techniques for the stabilization of accelerator magnets; (3) Predict the time-dependent luminosity performance of CLIC with the achieved magnet stability. These items are addressed in the Sections 2, 3 and 4, respectively. In Section 5 some conclusions are drawn.

\section{MEASUREMENT SETUP}

A detailed summary on the sensors used for vibration measurements in the framework of the CLIC Stability Study is given in [2]. Here, the main characteristics of

\footnotetext{
* PhD student at the University of Lausanne, CH, High Energy Physics Institute (UNIL-IPHE).
}
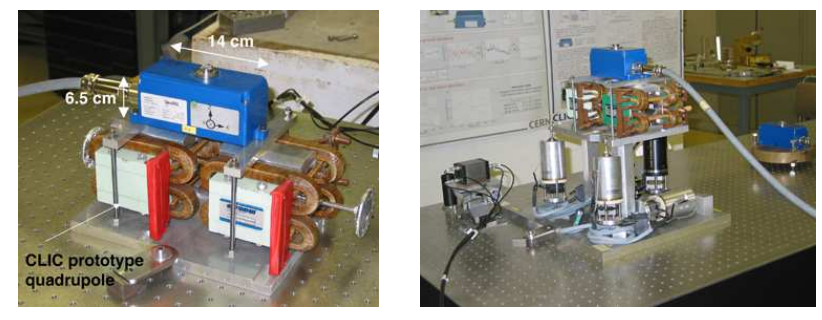

Figure 1: Installation of a CLIC prototype quadrupole on the stabilized honeycomb support structure. A doublet without (left) and with (right) a non vibration-optimized alignment support was used.

the sensors and the basic notation are briefly reviewed. The vibration measurements are performed with tri-axial geophones that measure velocities in the $4 \mathrm{~Hz}-315 \mathrm{~Hz}$ frequency range. In Fig. 1 two geophones installed on CLIC prototype quadrupoless are shown. These sensors have a sub-nanometer accuracy in the frequency range of interest. An upper limit for the resolution is given by the difference signal from two sensors placed side-by-side. At $4 \mathrm{~Hz}$ a $0.34 \mathrm{~nm}$ resolution was measured. The confidence in the absolute calibration of the geophones was assessed by comparing different vibration sensors. Two geophones from other manufacturers available at CERN and at ESRF [3] and a capacitive distance meter (relying on a different physical phenomenon for the vibration measurements) have been compared with the geophones of Fig. 1. These comparisons have demonstrated a good agreement between the different sensors. In a wide range of frequencies $(5 \mathrm{~Hz}-$ $50 \mathrm{~Hz})$ and vibration amplitudes $(0.1 \mathrm{~nm}-80 \mathrm{~nm})$ the various measurements show a spread of a few percent. On the basis of these results, the absolute error of the vibration measurements is believed to be smaller than $10 \%$.

A geophone provides a measure of the vibration velocity $v(n)$ at the discrete times $n \Delta t$, where $\Delta t=0.001 \mathrm{~s}$ is the sampling time and $n=1,2, \ldots, N . N$ is the total number of measurement points. The power spectral density of the displacement, $P\left(f_{k}\right)$ is defined for the discrete frequency $f_{k}=\frac{k}{N \Delta t}$ as:

$$
P\left(f_{k}\right)=\frac{N \Delta t^{3}}{2 \pi^{2} k^{2}}\left|\sum_{n=1}^{N} v(n) e^{-2 \pi i \frac{k n}{N}}\right|^{2} .
$$

The integrated RMS displacement induced by vibrations above $f_{\min }=\frac{k_{\min }}{N \Delta t}$ is given by:

$$
I\left(f_{\text {min }}\right)=\sqrt{\frac{1}{N \Delta t} \sum_{k^{\prime}=k_{\min }}^{k_{\max }} P\left(f_{k^{\prime}}\right)},
$$




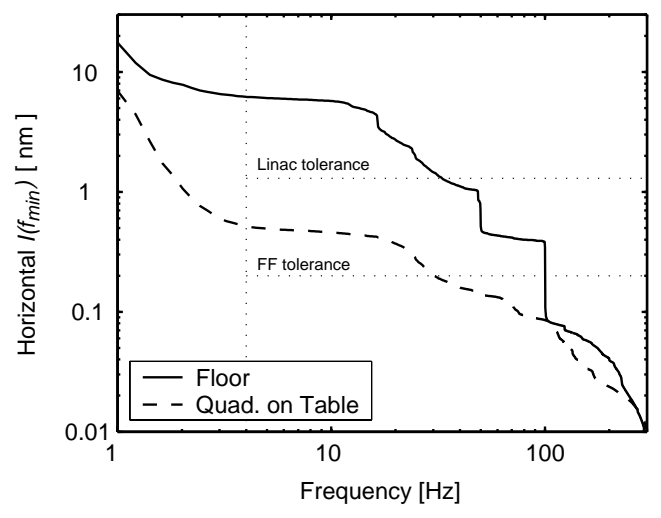

Figure 2: Integrated RMS vertical motion on the floor and on a stabilized CLIC prototype quadrupole(see Fig. 1, left part). Relevant CLIC tolerances are also indicated.

where $k_{\max }$ corresponds to the largest measurable frequency. In order to reduce the statistical uncertainty on the measurement results, $P\left(f_{k}\right)$ is calculated as the average of several consecutive data sets.

\section{QUADRUPOLE STABILIZATION TO THE SUB-NM LEVEL}

The stabilization of the CLIC prototype quadrupoles is performed with state-of-the-art stabilization devices. The magnets are fixed on top of an honeycomb support structure (table) that sits on a stabilizing system. The quadrupole doublets can either be directly screwed onto the table top or be installed on the table via an alignment support structure, which is a non vibration-optimized support used in CLIC Test Facility II (CTF2) for the micrometric alignment of the quadrupoles (see Fig.1). This setup has also been used for detailed studies of water induced vibrations [4], support structure resonances [2] and effects of acoustical waves. A soft pneumatic system and a piezo-based stiff system have been used for the stabilization of the doublet. Here, only the results achieved with the stiff system are considered. A more complete overview of both systems is given in [2].

The vertical and the horizontal RMS motion, as measured on the floor and on a quadrupole doublet, are shown in Figs. 2 and 3. These results were obtained with the experimental setup of Fig. 1, left part. Above $4 \mathrm{~Hz}$ the doublet was stabilized vertically to $(0.52 \pm 0.01) \mathrm{nm}$ with a ground motion of $(6.20 \pm 0.22) \mathrm{nm}$. The quadrupole vibration is within the CLIC linac tolerance $(1.3 \mathrm{~nm})$ and is only a factor 2.5 larger than the Final Focus tolerance $(0.2 \mathrm{~nm})$. The horizontal motion above $4 \mathrm{~Hz}$ was $(0.84 \pm 0.04) \mathrm{nm}$ for the quadrupole instead of $(3.05 \pm 0.07) \mathrm{nm}$ on the floor, both within the CLIC tolerances. The longitudinal motion on the quadrupole was $(4.33 \pm 0.28) \mathrm{nm}$ instead of $(4.30 \pm 0.08) \mathrm{nm}$ on the floor. This direction is not critical for luminosity considerations. It is important to point out that this magnet stability was achieved in a normal working environment of the CERN Meyrin site. The impact of measured quadrupole motion on the CLIC luminosity is discussed in the next section.

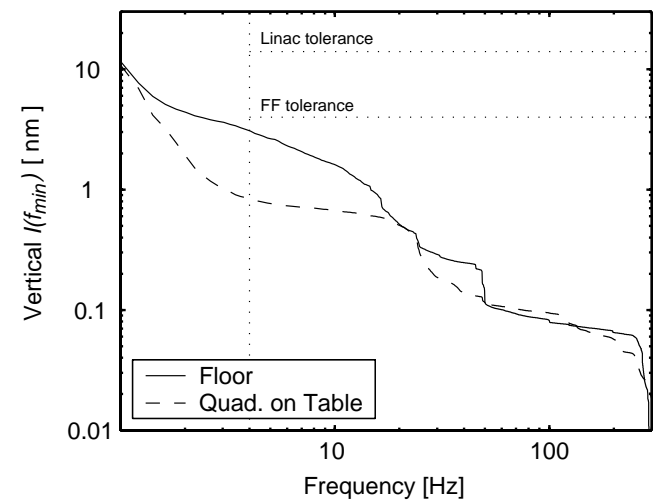

Figure 3: Integrated RMS horizontal motion on the floor and on a stabilized CLIC prototype quadrupole (see Fig. 1, left part). Relevant CLIC tolerances are also indicated.

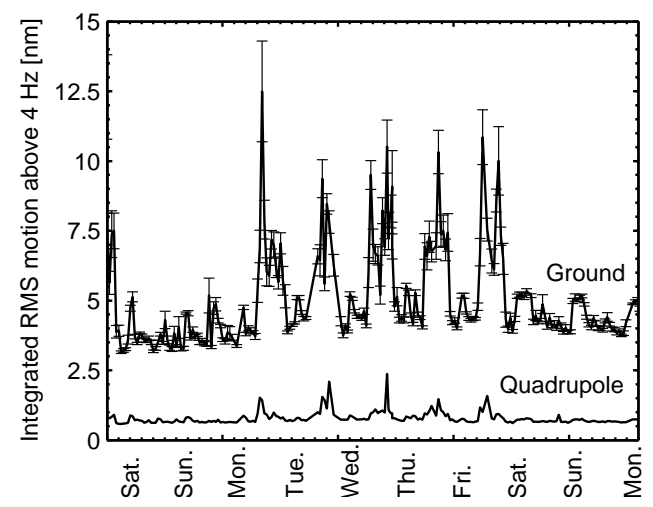

Figure 4: Vertical motion above $4 \mathrm{~Hz}$ versus time as measured on the floor and on a doublet, which was mounted on a CTF2-like alignment support. The error bars give the statistical uncertainty $(1 \sigma)$ and are omitted in the quadrupole lines because they are too small.

The quadrupole stability was continuously monitored for several days. On the table top, two doublets and one triplet were mounted on three independent alignment structures (see Fig. 1), like on a girder. The vertical motion above $4 \mathrm{~Hz}$ as measured on the floor and on one doublet is shown in Fig. 4 versus time. The cultural noise, which increases the ground motion up to $12 \mathrm{~nm}$ in the working days, is greatly reduced by the stabilization device: a stability below the $1 \mathrm{~nm}$ level is ensured almost independently of the cultural noise. Some residual perturbations are induced by people walking by the experimental setup or working close to it.

\section{CLIC LUMINOSITY PERFORMANCE}

The simulations of the time-dependent luminosity performance of CLIC have been performed for the beam delivery system (BDS) of [5], which provides a design luminosity of $0.8 \times 10^{35} \mathrm{~cm}^{-2} \mathrm{~s}^{-1}$. The validity of the tracking results has been assessed in $[6,7]$. The available codes agree within a few percent for simulations without synchrotron radiation, like the ones considered in the following. On the basis of quoted results, Merlin [8] was used for the 


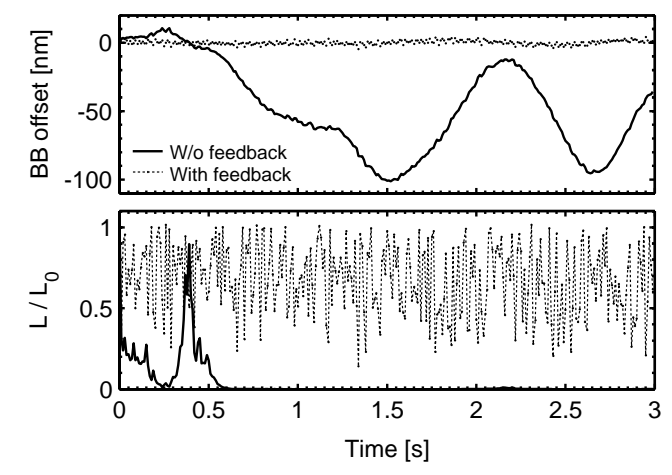

Figure 5: Time-dependent offset and luminosity for three seconds of CLIC operation with vibrating final doublets.

particle tracking. Full beam-beam (BB) simulations at the interaction point (IP) were performed with GuineaPig [9] and provided the luminosity and the BB deflection angle.

Two-beam simulations are carried out with the measured vibrational spectra of the CLIC prototype quadrupoles. The spectra are used to generate time-dependent misalignments of the quadrupoles at either side of the IP. Only the final doublets are moved because they induce the main impact on the luminosity (an additional $10 \%$ luminosity reduction is found if all other quadrupoles of the BDS are moved according to the same vibrational spectrum). A pulse-topulse feedback system for correcting the IP beam offset has been implemented in Merlin. The relative BB offset at the IP (to be minimized by the feedback) is calculated from the BB deflection angle provided by the GuineaPig simulations. Beam scans in the horizontal and vertical plane are used to calibrate the deflection angle versus beam offset. This curve is fitted and inverted to provide the offset from the beam angle, as was done for instance for SLC [10].

A result of a time-dependent simulation with vibrating quadrupoles is shown in Fig. 5. The BB offset (top) and the luminosity (bottom) versus time are given. This simulation uses the measurements of Fig. 2 to generate the quadrupole offsets (aligned machines at time zero). The motion of the two final doublets is taken to be uncorrelated. Without an IP feedback the BB offset would increase by several tens of nanometer and the luminosity would be completely lost within a few bunches. The feedback correction compensates the low frequency component of the motion and allows recovering a large fraction of the nominal luminosity.

A scan of the feedback gain has been done to find the set point that gives the best luminosity. Fig. 6 shows the average luminosity over three seconds of CLIC operation, i.e. 300 consecutive pulses. The quadrupole stability achieved with only the table as support allows recovering $66 \%$ of the CLIC design luminosity, to be compared with the $15 \%$ obtained if the quadrupoled move like the ground. If the motion of a stabilized doublet with its non vibrationaloptimized alignment support is considered, then $53 \%$ of the design luminosity is obtained. The horizontal motion has shown not to be critical for CLIC. Both with the horizontal vibrations measured on the floor and on the CLIC prototype quadrupoles (with or without alignment support)

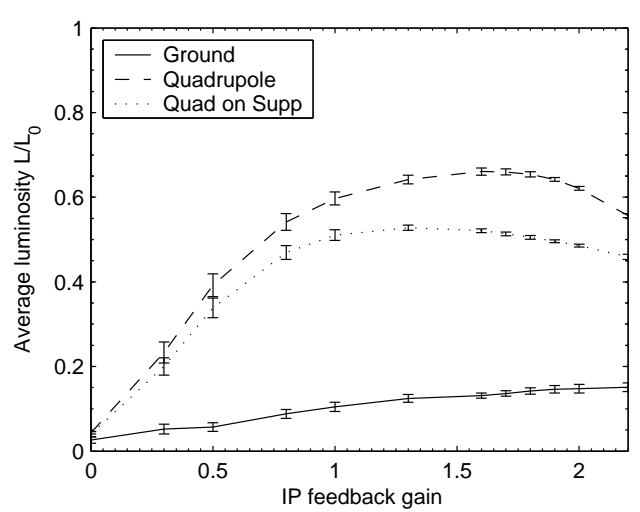

Figure 6: Average luminosity versus feedback gain when the doublets are moved according to the spectra of floor, quadrupole and quadrupole on an alignment support.

more than $95 \%$ of the design luminosity can be recovered with the IP feedback (for zero vertical motion).

\section{CONCLUSIONS}

For the first time a CLIC prototype quadrupole was stabilized vertically to the $0.5 \mathrm{~nm}$ level. This was achieved in a normal working area at CERN. This sub-nanometer stability allows ensuring that almost $70 \%$ of the CLIC design luminosity can be achieved. The horizontal and longitudinal vibrations of the stabilized quadrupole have proven not to be a critical issue for CLIC. Even though some further improvements are still required, these results demonstrate the basic feasibility of colliding nanobeams for CLIC.

The authors would like to acknowledge the help from the colleagues at DESY, SLAC and ESRF, all the members of the CERN CLIC team (in particular W. Schnell), F. Ruggiero, M. Mayoud.

\section{REFERENCES}

[1] G. Guignard et al., "A $3 \mathrm{TeV} \mathrm{e}^{+} \mathrm{e}^{-}$Linear Collider Based on the CLIC Technology," CERN-2000-008.

[2] R. Aßmann et al., "The CLIC Stability Study on the Feasibility of Colliding High Energy Nanobeams," Proc. Nanobeam2002, September 2-6, Lausanne, CH (2002).

[3] S. Redaelli, L. Zhang et al., to be published.

[4] S. Redaelli et al., "Status of the CLIC Studies on Water Induced Quadrupole Vibrations", Proc. Nanobeam2002, September 2-6, Lausanne, CH (2002).

[5] F. Zimmermann et al., "CLIC Beam Delivery System," Proc. Nanobeam2002, September 2-6, Lausanne, CH (2002).

[6] S. Redaelli et al., "Comparison of Simulation Tools for Beam Delivery Systems of Linear Colliders", EPAC2002, Paris, FR (2002).

[7] S. Redaelli et al., "Comparative Assessment of Simulation Tools for Beam Delivery Systems of Linear Colliders", Proc. Nanobeam2002, September 2-6, Lausanne, CH (2002).

[8] http://www.desy.de/ merlin/

[9] D. Schulte, "Beam-Beam Simulations with GUINEA-PIG," ICAP98, Monterey, CA., USA (1998).

[10] P. Bambade et al., Phys. Rev. Lett. 62, 2949 (1989). 
